\title{
The Prevalence and Risk Factors of Occult Stress Urinary Incontinence in Women Undergoing Genitourinary Prolapse Surgery
}

\author{
Jibril $\mathrm{AH}^{1}$, Ab Latip $\mathrm{N}^{2}(\bowtie), \mathrm{Ng}^{\mathrm{P}} \mathrm{Y}^{2}$, Jegasothy $\mathrm{R}^{3}$ \\ ${ }^{1}$ Department of Obstetrics and Gynaecology, Hospital Sultan Haji Ahmad Shah, Jalan Paya Luas, 28000 \\ Temerloh, Pahang, Malaysia. \\ ${ }^{2}$ Department of Obstetrics and Gynaecology, Hospital Kuala Lumpur, Jalan Pahang 50586, Wilayah \\ Persekutuan Kuala Lumpur, Malaysia. \\ ${ }^{3}$ Faculty of Medicine, MAHSA University, Jalan Elmu, Off Jalan Universiti, 59100, Kuala Lumpur, \\ Malaysia.
}

\begin{abstract}
De novo stress urinary incontinence (SUI) may occur in up to $80 \%$ of clinically continent women following genitourinary prolapse surgery. This had resulted in an increase in the rate of concurrent continence surgery during prolapse repair from 38\% in 2001 to $47 \%$ in 2009 in the United States. To date, there is no local data available to estimate the prevalence of occult SUI (OSUI) among Malaysian women awaiting surgery. Therefore, this study was conducted to elicit the prevalence of occult SUI and its associated risks factors in patients awaiting prolapse surgery. We retrospectively studied the records of 296 consecutive women with significant pelvic organ prolapse awaiting reconstructive repair. All patients attended the Urogynaecology Unit in Hospital Kuala Lumpur Malaysia between October 2007 and September 2011. They had undergone standardized interviews, clinical examinations and urodynamic studies. During the urodynamic testings, all prolapses were reduced using ring pessaries to elicit OSUI. Primary outcome was the prevalence of OSUI with prolapse reduction to predict possibility of developing de novo SUI following prolapse surgery. Secondary outcome was the assessment of potential risk factors for OSUI. Among the 296 women studied, 121 (40.9\%) were found to have OSUI. The risk factors associated with OSUI included age, BMI, numbers of SVD, recurrent UTI, reduction of urinary flow symptoms and grade 2 to 4 central compartment prolapses. We concluded that preoperative urodynamic testing with reduction of prolapse is useful to identify women with OSUI. This is important for preoperative counselling as well as planning for one step approach of prophylactic concomitant anti-incontinence procedures during prolapse surgery in order to avoid postoperative de novo SUI.
\end{abstract}

Keywords: pelvic organ prolapse, postoperative complications, reconstructive surgical procedure, stress urinary incontinence, urodynamics

\section{Correspondence:}

Norlelawati Ab Latip, Jabatan Obstetriks dan Ginekologi, Hospital Kuala Lumpur, Jalan Pahang 50586, Wilayah Persekutuan Kuala Lumpur, Malaysia. Tel: 03-26155450/56458 Fax: 03-26989845 Email: nabdullatip@yahoo.com

\section{Introduction}

Female pelvic organ prolapse is a major health issue affecting women's quality of life. It is estimated that up to $50 \%$ of women worldwide have some degree of genitourinary prolapse (1). The likelihood of prolapse surgery in their lifetime is reported to be greater than $10 \%$ by the age of 80 (2). In addition, the rate of concurrent prolapse repair and continence procedures appears to be increasing. Data from the United States National Inpatient study revealed an increment in the percentage of concurrent continence surgery for apical prolapse repair procedures, from $38 \%$ in 2001 to $47 \%$ in 2009 (3). This could be attributed to the increase in the incidence of de novo postoperative stress urinary incontinence (SUI). 
De novo SUI after prolapse corrective surgery is affecting $36 \%$ to $80 \%$ of clinically continent women with advanced prolapse (4). OSUI is elicited when a continent woman has a positive cough test on prolapse reduction during urodynamic study (5). Urodynamic diagnostic criteria include leakage with straining in the absence of detrusor contraction or a decreased in maximal urethral closure with prolapse reduction (4).

The mechanism by which severe prolapse particularly large cystocele may mask SUI in an apparently continent women is via the cushioning effect of the cystocele which dissipated the effect of the abdominal pressure on the bladder outlet. In addition, the prolapsed bladder on the urethra may also cause urethral kinking causing an increase in the intra-urethral pressure (6). As for posterior wall defect, the prolapsed bowel may compress and/or support the bladder neck, providing continence during straining. Corrective prolapse surgery may reverse this mechanism, rendering these women to develop postoperative SUI. In contrast, overcorrection may lead to voiding dysfunction (7). Therefore, preoperative detection of OSUI may justify the indication for concomitant prophylactic antiincontinence procedure during prolapse surgery in women at risk (1).

History of preoperative SUI is identified as the sole independent predictor of subjective (RR 4.03) and objective SUI (RR 4.67; 95\%CI) and subsequent antiincontinence surgery (RR 6.17) after laparoscopic prolapse surgery (sacrocolpopexy) in patient with negative occult stress testing (8). In another study by the Belgium group, it was found that no statistical association existed between clinical data prior to operation (age, body mass index, maximum urethral closure pressure, medical history of urge urinary incontinence (UUI) and prolapse surgery, bladder neck hypermobility) and de novo SUI after surgery (9).

Currently, there are no local data in Malaysia to estimate the prevalence and predict the risks factors of OSUI among Malaysian women with pelvic organ prolapse. Thus, the aim of this study is of two fold, to detect the prevalence of OSUI in Malaysia using a larger sample size than previous studies and to determine the risk factors of OSUI.

\section{Materials and Methods}

This was a retrospective, cross-sectional study spanning a period of 4 years from October 2007 to September 2011, of 296 women with significant pelvic organ prolapse awaiting vaginal reconstruction surgery. The records of the women who attended the outpatient in Urogynaecology clinic of Department of Obstetrics and Gynaecology, Kuala Lumpur Hospital were reviewed.
The subjects were asymptomatic of SUI, but had bothersome prolapse symptoms that were staged according to Pelvic Organ Prolapse Quantification System of the International Continence Society (ICS POP-Q). The study was approved by the Institutional Review Board of Hospital Kuala Lumpur. The women were excluded if they presented with preoperative urinary incontinence, concurrent pelvic disease requiring laparotomy, previous pelvic surgery and/or history of anti-incontinence procedure.

The participants underwent a routine preoperative assessment that includes a standardized interview, clinical examination, free flowmetry and multichannel urodynamic testing. All women had reduction of prolapse using a well-fitting ring pessary, making sure that there was no urethral compression. Multichannel urodynamic were done according to the recommendations of the International Continence Society.

The observation of SUI during urodynamics (urodynamic SUI) is defined as the finding of involuntary leakage during filling cystometry associated with increased intra-abdominal pressure in the absence of a detrusor contraction. The symptom of SUI is the complaint of involuntary loss of urine on effort or physical exertion (e.g., sporting activities) or on sneezing or coughing. This is to be differentiated from the sign of SUI, which is the observation of involuntary leakage from the urethra synchronous with effort or physical exertion or on sneezing or coughing.

Urodynamic SUI was defined as unintentional leakage of urine upon valsalva manoeuver in the absence of simultaneous detrusor contraction, observed in patients without prolapse reduction during urodynamic testing. These patients were excluded in this study. On the other hand, urodynamic OSUI was defined as uncontrolled escape of urine diagnosed during a rise in the abdominal pressure, without modification in the detrusor pressure following prolapse reduction. The findings of urodynamic tests in this study were divided into two:

1. negative/normal if no urine leakage observed with prolapse reduction

2. Positive/urodynamic OSUI with presence of leakage on prolapse reduction.

The main outcome measures were the prevalence of OSUI and its associated risks factors. The data were analysed statistically using the simple and multiple logistic regression for individual variables and multiple variables respectively. Analysis was done using SPSS programs with $\mathrm{p}<0.05$ considered as statistically significant. The study was approved by the Clinical 
Research Centre, Kuala Lumpur Hospital and Malaysian Science University ethics committee.

\section{Results}

A total of 296 patients were recruited into this study. In general, voiding function was improved after prolapse reduction with a ring pessary. Of the 296 continent women, 121 (40.9\%) had demonstrable urodynamic OSUI with prolapse reduction; the remaining 175 women $(59.1 \%)$ had negative findings (Table 1$)$. The mean age of the women studied was $58.60 \pm 8.7$ years and the median parity was 3 (Table 2). Majority of them were Malay ( $\mathrm{n}=162 ; 54.7 \%)$, followed by Indian $(\mathrm{n}=75$; $25.3 \%)$ and Chinese $(n=55 ; 18.6 \%)$. The mean BMI of the participants was $26.18 \mathrm{~kg} / \mathrm{m}^{2}$ (range 20 to 34.9).Two hundred and twenty-four (75.7\%) were postmenopausal and 72 (24.3\%) were either pre or perimenopausal; 43 (19.2\%) of the postmenopausal women were on HRT (Table 2). According to the International Continence Society Pelvic Organ Prolapse Quantification System, significant prolapse was defined as prolapse occurring at stage 2 or greater. Most of our patients had a mixture of 2 or 3 compartments prolapse. Of the 296 women, 269 (90.9\%) and 250 (84.6\%) had significant cystocele and rectocele respectively. Two hundred and sixty-two (88.5\%) were diagnosed with significant central compartment prolapse. For each significant compartment prolapse (stage 2 to 4), majority of them had stage 2 prolapse with 109 patients each had cystocele and utero-vaginal prolapse and 160 patients with rectocele.

A preoperative finding of OSUI is generally accepted as a marker for increased likelihood of postoperative SUI after prolapse repair. Using multiple logistic regression, our study showed significant association between age, body mass index, parity, number of vaginal delivery, history of recurrent urinary tract infections and reduced preoperative urinary flow with the development of OSUI (Table 3). In contrast with advanced anterior and posterior compartment prolapse, significant (stage 2 -4) central compartment prolapse was identified as a risk factor for OSUI. More than $80 \%$ of women who were diagnosed with OSUI in our study had at least grade 2 or more of pelvic organ prolapse. Variables such as parity, menopausal status and history of dysuria were not significantly associated with OSUI after controlling for the confounders. No association was found between ethnicity, smoking status, history of instrumental delivery, chronic medical illness and OSUI in this study.

Table 1: Preoperative Urodynamic Occult Stress Urinary Incontinence Result

\begin{tabular}{cc}
\hline Test result & No of patients (\%) \\
\hline Positive & $121(40.9 \%)$ \\
Negative & $175(59.1 \%)$ \\
Total & $296(100 \%)$ \\
\hline
\end{tabular}

Table 2: Characteristics of women participated in this study $(n=296)$

\begin{tabular}{|c|c|c|c|}
\hline Variable & & & Mean (SD) \\
\hline Age, mean \pm SD $(y r)$ & & & $58.60 \pm 8.7$ \\
\hline Body Mass Index (kg/m2) & & & $26.18 \pm 4.16$ \\
\hline Parity (median) & & & 3 \\
\hline \multicolumn{4}{|l|}{ Ethnicity } \\
\hline Malay & & & $154(52)$ \\
\hline Indian & & & 94 (31.8) \\
\hline Chinese & & & 44 (14.9) \\
\hline Others & & & $4(1.4)$ \\
\hline \multicolumn{4}{|l|}{ Marital status } \\
\hline Married & & & $237(80.1)$ \\
\hline Unmarried/ Widower/Divorced & & & 59 (19.9) \\
\hline \multicolumn{4}{|l|}{ Menopausal status } \\
\hline Postmenopausal & & & $224(75.7)$ \\
\hline HRT & & & $43(19.2)$ \\
\hline No HRT & & & $181(80.8)$ \\
\hline Pre/perimenopausal & & & $72(24.3)$ \\
\hline Stage of Prolapse Presentation & Cystocele & Rectocele & UV prolapse \\
\hline Stage 0 & $3(1.0)$ & $4(1.4)$ & $10(3.4)$ \\
\hline Stage I & $24(8.1)$ & $42(14.2)$ & $24(8.1)$ \\
\hline Stage II & $109(36.8)$ & $160(54.1)$ & $109(36.8)$ \\
\hline Stage III & $83(28.0)$ & $28(9.5)$ & $60(20.2)$ \\
\hline Stage IV & $77(26.0)$ & $62(20.9)$ & $93(31.4)$ \\
\hline
\end{tabular}


Table 3: Table showing association of different variables with occult SUI

\begin{tabular}{|c|c|c|}
\hline Variable & Crude Hazard Ratio (95\% CI) & P value \\
\hline Age & $1.044(1.001-1.090)$ & $<0.046^{*}$ \\
\hline BMI & $1.151(1.078-1.228)$ & $<0.001^{*}$ \\
\hline Ethnicity & - & 0.999 \\
\hline Menopausal status & $1.901(0.714-5.060)$ & 0.199 \\
\hline HRT user & $0.844(0.418-1.700)$ & 0.634 \\
\hline Smoking status & $0.737(0.351-1.549)$ & 0.421 \\
\hline Parity & $1.214(1.086-1.352)$ & $<0.001^{*}$ \\
\hline \multicolumn{3}{|c|}{ History of Mode of Delivery } \\
\hline SVD & $1.216(1.086-1.361)$ & $<0.001 *$ \\
\hline Forceps & $0.845(0.366-1.949)$ & 0.693 \\
\hline Vacuum & $0.618(0.280-1.366)$ & 0.234 \\
\hline LSCS & $0.737(0.351-1.549)$ & 0.421 \\
\hline \multicolumn{3}{|l|}{ Medical condition } \\
\hline Diabetes mellitus & $0.933(0.559-1.555)$ & 0.790 \\
\hline Hypertension & $0.693(0.412-1.165)$ & 0.167 \\
\hline Stroke & $0.377(0.033-4.324)$ & 0.433 \\
\hline Recurrent UTI & $2.578(1.208-5.498)$ & $0.014^{*}$ \\
\hline \multicolumn{3}{|l|}{ Urinary symptoms } \\
\hline Frequency & $0.290(0.150-5.536)$ & 0.411 \\
\hline Nocturia & $0.479(0.043-5.332)$ & 0.549 \\
\hline Reduced flow & $0.217(0.100-0.471)$ & $<0.001 *$ \\
\hline Dysuria & 9.078 (1.079-76.397) & 0.420 \\
\hline \multicolumn{3}{|l|}{ Stage of Prolapse } \\
\hline \multicolumn{3}{|l|}{ Cystocele } \\
\hline Stage I & 0.000 & 0.999 \\
\hline Stage II & 0.000 & 0.999 \\
\hline Stage III & $0.531(0.146-1.938)$ & 0.338 \\
\hline Stage IV & $1.443(0.438-4.756)$ & 0.547 \\
\hline \multicolumn{3}{|l|}{ Rectocele } \\
\hline Stage I & $14.019(0.419-46.94)$ & 0.141 \\
\hline Stage II & 0.179(0.029-1.089) & 0.062 \\
\hline Stage III & $0.290(0.084-1.003)$ & 0.051 \\
\hline Stage IV & $0.315(0.071-1.396)$ & 0.127 \\
\hline \multicolumn{3}{|l|}{ UV Prolapse } \\
\hline Stage I & 0.000 & 0.999 \\
\hline Stage II & $0.320(0.02-0.484)$ & $0.013^{*}$ \\
\hline Stage III & $0.101(0.031-0.328)$ & $<0.001 *$ \\
\hline Stage IV & $1.025(0.354-2.970)$ & $0.03 *$ \\
\hline
\end{tabular}

Abbreviations: SUI, stress urinary incontinence; BMI, body mass index; HRT, hormone replacement therapy; SVD, spontaneous vertex delivery; LSCS, lower segment caesarean section; UV, uterovaginal. Based on multiple logistic regression, asterisk indicates significant $\mathrm{p}$ value $<0.05$. 


\section{Discussion}

Occult stress urinary incontinence (OSUI) is the presence of involuntary urinary leakage on effort or exertion which is observed following reduction of coexisting prolapse. It has been called "masked", "potential", "latent", hidden" or "reduced" stress urinary incontinence in the literature, prior to the standardization of the female pelvic floor dysfunction (10). The true incidence of occult stress urinary incontinence is not clear and results varied considerably between studies. This may be due to lack of standardized test and different methods used to diagnose occult stress urinary incontinence. A wide range of incidence was reported from $6 \%$ to $80 \%$ (11) with clinical diagnosis and about $27 \%$ with cystometry (12). Others used pressure transmission ratio as an indicator for OSUI, with incidences ranging from 36 to $73 \%$ (13).

The need for preoperative screening for OSUI cannot be overstated. A Cochrane meta-analysis had shown that omitting continence surgery during prolapse repair in women with OSUI increased the risk of postoperative SUI by 2.2 (CI 1.4 to 3.3) (14). In addition, prophylactic anti incontinence procedure was both effective and safe in patients with OSUI undergoing prolapse repair (15). However, it was associated with de novo detrusor over activity (7.6\%), urinary tract infections (7.6\%) and urinary retention (3.8\%) (16). Despite fear of morbidity, adding prophylactic continence surgery may be justified as it is a one-step approach to solve two problems. Furthermore, continence surgery at the time of prolapse operation had shown to prevent $25 \%$ of the women from developing postoperative SUI (14).

Previous studies had published prevalence of OSUI ranging from $22-87 \%$. This is in agreement with our study which showed prevalence of $40.9 \%(\mathrm{n}=121)$. In contrast, Reena et al. (17) and Rosenzweig et al. (18) found higher prevalence of OSUI of $67.9 \%$ and 59\% respectively. This may be attributed to more patients with advanced prolapse (stage 3 and above) were recruited in their studies compared to our study (mainly stage 2 and above). Nevertheless, more than $80 \%$ of our subjects diagnosed with OSUI had advanced pelvic organ prolapse (stage 3 and 4) in all 3 compartments, highest occurrence was found in patients with stage 4 cystocele. These results were supported by several studies which found mainly severe degree of pelvic organ prolapse masked SUI in clinically continent women and those women were at higher risk of developing postoperative SUI $(5,14,15,16)$.

Our prevalence rates of OSUI was higher compared to those found in CARE trial (40.9\% vs. 19\%) (6). This could be explained by the difference in repositioning method used. Despite various methods of prolapse reduction used in the CARE trial, the detection rate of OSUI was lower compared to our study which only use ring pessary as the reduction method (6). Other authors used rectal swabs, Gel horn pessary or Grave's speculum for prolapse reduction (17). Prolapse repositioning with rectal swabs was superior in detecting OSUI as it significantly lowered the midurethral closure pressure and causes a shorter functional urethral length compared to other techniques. This is because low MUCP and functional urethral length during preoperative urodynamic evaluation are significant predictors for occult SUI (19). In contrast, another consensus showed detection rate was lower with pessary than with speculum prolapse reduction (20).

Although pessaries are commonly used for prolapse reduction and predictive of postoperative stress urinary incontinence (4), combination of repositioning tests (manual, pessary in the office, or pessary continuous use, or combination of two or all) are thought to be more predictive in diagnosing occult stress urinary incontinence than using a single test alone (4). Unfortunately as ring pessary is the only method used in our study, we were not able to compare the use of various reduction methods. Nevertheless, our detection rate is almost similar with Reena et al. whose used ring pessary reduction technique with prevalence rate of $59 \%$ (17). Ambulatory pessary test conducted over several days is another alternative technique to clinical examination as it allows an optimal continence screening during the patient's normal daily life. However, there are limitations to this test namely unwillingness of some women or failure of pessary fitting for several reasons (1).

A study by Chiarelli et al. reported that the prevalence rate of stress urinary incontinence among elderly Australian females aged 70-75 years was 35\% (21). In another study conducted in Norway (EPICONT Study) which involved over 20,000 women, the rate of incontinence in women aged 50 was $30 \%$ and steadily increased until age of 70 (22). Similarly, older women are at higher risk of developing OSUI (17). This is in accordance with our findings which suggested a significant association between age and OSUI (Crude Hazard Ratio 1.044 (1.001 - 1.090), $\mathrm{p}<0.046)$. Occult SUI was diagnosed in 11 patients (3.7\%) below the age of 50, in our study. Women aged 50 years or older had twice the risk of OSUI compared to those younger than 50 years $(\mathrm{P}<0.005)$. This is comparable to Lo et al. who found that women older than 66 years were 2.86 times at greater risk of developing de novo SUI postoperatively (19). In addition, age is an important 
confounding factor in relation to concomitant medical illnesses which may aggravate urinary incontinence.

We found a significant correlation between body mass index (BMI) and OSUI (Crude Hazard Ratio 1.151 (1.078 - 1.228), $\mathrm{p}<0.001)$. The prevalence of OSUI was higher among the obese (BMI $>35 \mathrm{~kg} / \mathrm{m} 2)$ compared to the overweight group (BMI $>30 \mathrm{~kg} / \mathrm{m} 2$ ), with the former $74 \%$ versus the latter $48 \%(p<0.05)$. The most probable explanation for this is an increase in BMI resulted in an increase in intra-abdominal and intravesicular pressure. This will increase the risks of urethral kinking and subsequently favours the development of OSUI. Reversing this effect by weight loss would significantly improve incontinence in obese women (23). Our data showed similar result with only $24 \%$ of female subject with normal BMI had OSUI.

Previous literature was inconsistent in describing the role of menopause and estrogen deficiency in urinary incontinence. Reena et al. found postmenopausal women had a statistically significant risk of developing OSUI (17). This is supported by Lo et al. who showed postmenopausal status is independent predictors for de novo SUI following extensive POP repair (19). In addition, estrogen replacement is thought to alleviate incontinence by facilitating proliferation and maturation of urethral mucosa in postmenopausal women (24). Our study also found the risk of OSUI was increased in postmenopausal women and decreased with hormone replacement therapy. However, these associations were insignificant (Table 3).

Our study demonstrated an increased risk of OSUI among women who had vaginal delivery compared to other mode of deliveries $(\mathrm{p}<0.001)$. A further increase was observed among multiparous women with parit

$7(p<0.001)$. These results may suggest that repetitive mechanical strain during labour may add to the risk of SUI compared to pregnancy alone. This was supported by Rortveit et al. (25) who studied over 15,000 women and found an association between stress urinary incontinence and caesarean delivery compared to nulliparous women and more prevalent after a vaginal delivery compared to caesarean delivery (EPICONT study) (22). In addition, Dietz found an association between intra-partum event and development of urinary incontinence in later life (26).

We elicited significant associations between recurrent urinary tract infections (Crude Hazard Ratio 2.578 (1.208-5.498), $\mathrm{p}<0.014)$ and reduced urinary flow (Crude Hazard Ratio 0.217 (0.100-0.471), $\mathrm{p}<0.001)$ with presence of OSUI (Table 3). This was in agreement with previous study done by Gretchen et al. (27) who found that recurrent UTI causes over distension of the bladder due to bladder stasis which may damage the stretch receptors in the bladder wall. Bladder stasis is contributed by the kinking of urethra in prolapse, hence predisposed women to UTI. We also found a significant increase in detection of occult SUI with an increase in the staging of prolapse particularly in stages 3 (Crude Hazard Ratio 0.101 (0.031-0.328), $\mathrm{p}<0.001$ ). This may be explained by the degree of urethral kinking and compression as well as dissipation of pressure away from the bladder neck. This effect is relatively marked in advance prolapse thus increasing the incidence of OSUI (17).

The incidence of de novo postoperative OSUI in preoperatively asymptomatic continent women remained unclear following prolapse repair $(1,28)$. As the incidence of OSUI in this study is only a prediction of possible outcome following prolapse surgery, further larger series and longer prospective studies are needed to explore the true incidence and actual outcome of postoperative OSUI (29). Based on our data, we concluded that women should have a preoperative urodynamic screening for OSUI to identify those at risk of postoperative OSUI. We also found that parity, body mass index, history of spontaneous vertex delivery, history of reduced urinary flow and stage 3 uterovaginal prolapse were significant risk factors for OSUI $(\mathrm{p}<0.001)$. This information could be used to facilitate the development of a risk stratification system in the clinical setting in order to identify the target groups who are more likely to benefit from the preoperative urodynamics testing and have OSUI. Hence, strategic plans could be implemented in terms of patient's counselling, optimum surgery performed and possible postoperative complications anticipated.

In agreement with other study, we found urodynamic testing after restoring prolapse anatomy with a ring pessary is the preferable method as it may identify women needing anti-incontinence procedures during prolapse surgery (30).

\section{Conclusion}

Identifying risk factors of developing de novo OSUI as found in our study was essential for predicting the correct diagnosis. The decision whether to perform a combined surgery in either a one or two - step approaches requires weighing the possibility of inadequate treatment versus exposing the patient to the hazards of unwarranted surgery.

\section{References}

1. Al-Mandeel H, Al-Badr A. Management of occult stress urinary incontinence with 
prolapse surgery. Minerva Ginecol 2013; 65(4): 417-24.

2. Olsen AL, Smith VJ, Bergstrom JO, Colling JC, Clark AL. Epidemiology of surgically managed pelvic organ prolapse and urinary incontinence. Obstet Gynecol 1997; 89(4): 501-6.

3. Raman SV, Raker CA, Sung VW. Concomitant apical prolapse repair and incontinence procedures: trends from 20012009 in the United States. Am J Obstet Gynecol 2014; 211(3): 222.e1-5.

4. Haessler AL, Lin LL, Ho MH, Betson LH, Bhatia NN. Reevaluating occult incontinence. Curr Opin Obstet Gynecol 2005; 17(5): 53540.

5. Gordon D, Gold RS, Pauzner D, Lessing JB, Groutz A. Combined genitourinary prolapse repair and prophylactic tension-free vaginal tape in women with severe prolapse and occult stress urinary incontinence: preliminary results. Urology 2001; 58(4): 547-50.

6. Visco AG, Brubaker L, Nygaard L, et al. The role of preoperative urodynamic testing in stress-continent women undergoing sacrocolpopexy: the Colpopexy and Urinary Reduction Efforts (CARE) randomized surgical trial. Int Urogynecol J Pelvic Floor Dysfunct 2008; 19(5): 607-14.

7. Wei JT, Nygaard I, Richter HE, et al. A midurethral sling to reduce incontinence after vaginal prolapse repair. N Eng J Med 2012; 366(25): 2358-67.

8. Leruth J, Fillet M, Waltregny D. Incidence and risk factors of post-operative stress urinary incontinence following laparoscopic sacrocolpopexy in patients with negative preoperative prolapse reduction stress testing. Int Urogynecol J 2013; 24(3): 485-91.

9. Misrai V, Roupret M, Cour F, Chartier-Kastler E, Richard F. De novo urinary stress urinary incontinence after laparoscopic sacral colpopexy. BJU Int 2008; 101(5): 594-7.

10. Haylen B, de Ridder D, Freeman RM, et al. An International Urogynecological Association (IUGA)/International Continence Society (ICS) joint report on the terminology for female pelvic floor dysfunction. Neurourol Urodyn 2010; 29(1): 4-20.

11. Chaikin DC, Groutz A, Blaivas JG. Predicting the need for anti-incontinence surgery in continence women undergoing repair of severe urogenital prolapse. J Urol 2000; 163(2): 5314.

12. Hextall A, Boos K, Cardozo L, Toozs-Hobson P, Anders K, Khullar V. Videocystourethrography with a ring pessary in situ. A clinically useful preoperative investigation for continent women with urogenital prolapse? Int Urogynecol J Pelvic Floor Dysfunct 1998; 9(4): 205-9.

13. Bump RC, Norton PA. Epidemiology and natural history of pelvic floor dysfunction. Obstet Gynecol Clin North Am 1998; 25(4): 723-46.

14. Maher C, Feiner B, Baessler K, Schmid C. Surgical management of pelvic organ prolapse in women. Cochrane Database Syst Rev 2013; 4: CD004014.

15. Groutz A, Levin I, Gold R, Pauzner D, Lessing JB, Gordon D. "Inside-out" transobturator tension-free vaginal tape for management of occult stress urinary incontinence in women undergoing pelvic organ prolapse repair. Urology 2010; 76(6): 1358-63.

16. Liang CC, Chang YL, Chang SD, Lo TS, Soong YK. Pessary test to predict postoperative urinary incontinence in women undergoing hysterectomy for prolapse. Obstet Gynecol 2004; 104(4): 795-800.

17. Reena C, Kekre AN, Kekre N. Occult stress incontinence in women with pelvic organ prolapse. Int J Gynecol Obstet 2007; 97(1): 314.

18. Rosenzweig BA, Pushkin S, Blumenfeld D, Bhatia NN. Prevalence of abnormal urodynamic test results in continent women with severe genitourinary prolapse. Obstet Gynecol 1992; 79(4): 539-42.

19. Lo TS, Bt Karim N, Nawawi EA, Wu PY, Nusee Z. Predictors for de novo stress urinary incontinence following extensive pelvic reconstructive surgery. Int Urogynecol J 2015; 26(9): 1313-19. 
20. Fritel X, Fauconnier A, Bader G, et al. Diagnosis and management of adult female stress urinary incontinence: guidelines for clinical practice from the French College of Gynaecologists and Obstetricians. Eur J Obstet Gynecol Reprod Biol 2010; 151(1): 14-9.

21. Chiarelli P, Brown W, McElduff P. Leaking Urine: prevalence and associated factors in Australian women. Neurourol Urodyn 1999; 18(6): 567-77.

22. Hannestad YS, Rortveit G, Sandvik H, Hunskaar S. A community-based epidemiological survey of female urinary incontinence: the Norwegian EPICONT Study. Epidemiology of Incontinence in the County of Nord-Trøndelag. J Clin Epidemiol 2000; 53(11): 1150-57.

23. Subak LL, Johnson C, Whitcomb E, Boban D, Saxton J, Brown JS. Does weight loss improve incontinence in moderately obese women? Int Urogynecol J Pelvic Floor Dysfunct 2002; 13(1): 40-3.

24. Rioux JE, Devlin C, Gelfand MM, Steinberg WM, Hepburn DS. 17 beta-estradiol vaginal tablet versus conjugated equine estrogen vaginal cream to relieve menopausal atrophic vaginitis. Menopause 2000; 7(3): 156-61.

25. Rortveit G, Daltveit AK, Hannestad YS, Hunskaar S. Urinary incontinence after vaginal delivery or cesarean section. $\mathrm{N}$ Engl J Med 2003; 348(10): 900-7.
26. Dietz HP. Pelvic floor trauma following vaginal delivery. Curr Opin Obstet Gynecol 2006; 18(5): 528-37.

27. Gretchen M. Gynecology and Obstetrics 2003 on CD-ROM - Urinary Tract Infections in Obstetrics and Gynecology. Philadephia: Lippincott Williams \& Wilkins, 2004 (Vol 2, Chapter 43)

28. Kuribayashi M, Kitagawa Y, Narimoto K, Urata S, Kawaguchi S, Namiki M. Predictor of de novo stress urinary incontinence following TVM procedure: a further analysis of preoperative voiding function. Int Urogynecol J 2013; 24(3): 407-11.

29. van der Ploeg JM, van der Steen A, Oude Rengerink K, van der Vaart $\mathrm{CH}$, Roovers JP. Prolapse surgery with or without stress incontinence surgery for pelvic organ prolapse: a systematic review and meta-analysis of randomised trials. BJOG 2014; 121(5): 53747.

30. Sinha D, Arunkalaivanan AS. Prevalence of occult stress incontinence in continent women with severe genital prolapse. J Obstet Gynaecol 2007; 27(2): 174-6. 